\title{
The liquid-liquid flow dynamics and droplet formation in a modified step T-junction Microchannel
}

\author{
Jingwei Zhang ${ }^{1}$, Si Da Ling ${ }^{1}$, zhuo chen ${ }^{1}$, Wenjun $\mathrm{Ma}^{1}$, and jianhong $\mathrm{Xu}^{2}$ \\ ${ }^{1}$ Affiliation not available \\ ${ }^{2}$ the State Key Lab of Chemical Engineering
}

July 15, 2021

\begin{abstract}
The droplet generation mechanism in the step T-junction remains unknown, especially for the transition stage from dripping to jetting. In this work, the droplet generation mechanism was systematically investigated in a novel modified step T-junction. We found that under different fluid regimes, different factors take action. In dripping regime, the interfacial tension dominated the formation mechanism when the surfactant concentration was controlled below micelle concentration (CMC). In jetting regime, our experimental results showed that the influence of the surfactant concentration on the size of generated droplets was rather negligible while the phase ratio indeed determined such a parameter. In the dripping-jetting transition stage, an abnormal increase of droplet size was observed despite the increase of continuous phase flow. To the best for our knowledge, it is the first study to report generation mechanism in modified step T-junction from dripping to jetting regimes.
\end{abstract}

\section{Hosted file}

manuscript.docx available at https://authorea.com/users/425886/articles/530580-the-liquidliquid-flow-dynamics-and-droplet-formation-in-a-modified-step-t-junction-microchannel 